\title{
Transtornos mentais em pacientes com Fibromialgia
}

\author{
AUTORES: Luup, I.A; Rodrigues, L.F; Rossi, C.B \\ ORIENTADORA: Torres, T.M \\ INSTITUIÇÃO: Centro Universitário São Camilo \\ Email do Autor Principal: igor_augustoluup@hotmail.com
}

\section{Introdução}

A fibromialgia (FM) é uma doença comum caracterizada por dor generalizada de longa duração associada a fadiga, má qualidade do sono, distúrbios cognitivos, e sofrimento psicológico. Como critérios de diagnóstico tem-se dor por mais de três meses em todo o corpo e presença de pontos dolorosos na musculatura (11/18 pontos). O diagnóstico clínico e FM continua a ser um desafio, uma vez que os sintomas podem ser encontrados em outras doenças reumatológicas. A depressão e a ansiedade são os transtornos psiquiátricos mais comuns em pacientes com FM, $20-80 \%$ e 13-63\% dos pacientes, respectivamente. Através de escalas e escores, estudos avaliaram transtornos mentais em pacientes com FM, os quais demonstram que grande maioria dos portadores da doença não possuem qualidade de vida, devido à dor crônica, dificuldade de tratamento e muitas vezes, até mesmo, dificuldade no diagnóstico.

\section{Objetivo}

Avaliar a prevalência dos transtornos mentais na população com fibromialgia e o impacto gerado por eles no desenvolvimento da doença

\section{Metodologia}

A pesquisa foi realizada através da Biblioteca Virtual de Saúde integrada a LILACS, onde foram utilizadas as seguintes palavras chave: FM, transtorno de ansiedade, transtorno mental, transtorno depressivo e transtorno de humor. Foram incluídos estudos observacionais, estudo de prevalência, revisão sistemática e relato de caso. Após realizada a busca, foram encontrados 89 artigos, dos quais após seleção, conferência e exclusão, restaram 15 artigos selecionados para a realização do trabalho.

\section{Resultados}


Os estudos mostraram que grande parte dos pacientes avaliados são mulheres e acima da idade de 30 anos, apresentando mais frequentemente sintomas como descontentamento geral, tristeza, temperamento ansioso, distúrbios de sono e em alguns casos sintomas dissociativos.

Para depressão, as principais escalas usadas foram: Escala Hospitalar de Ansiedade e Depressão, Inventário de depressão de Beck (BDI) - versão Espanhola, Escala de Experiências Dissociativas (DES), Patient Health Questionnaire e The Personal Health Questionnaire (PHQ9); já para ansiedade utilizaram-se as seguintes escalas: Escala Hospitalar de Ansiedade e Depressão (HADS) e Escala de temperamento de Memphis, Pisa e San Diego - versão autoadministrada (TEMPS-A), Inventário de Ansiedade Traço Estadual (STAI) - versão Espanhola e Inventário de ansiedade de Beck (BAI).

O perfil de personalidade observado na FM é definido como sendo propenso a reagir a estressores com um aumento na ansiedade, tendo uma tendência a pensamentos negativos em resposta às frustrações do dia-a-dia e antecipação problemas futuros, apresentando alta fatigabilidade, e mostrando pouca habilidade para controlar e regular a seu comportamento para adaptar-se às situações, de acordo com o seus objetivos e valores próprios. Pessoas com esse perfil tendem a reagir com indiferença ou rejeição à novidades, e se sentirem confortáveis com a monotonia evitando as situações de risco. Essas características podem levá-los a fícarem desanimados quando confrontados com situações potencialmente indutoras de ansiedade.

Do ponto de vista fisiopatológico os baixos níveis séricos de serotonina, baixos níveis de metabólitos da serotonina no líquido cefalorraquidiano, norepinefrina e dopamina, e níveis mais elevados de cortisol plasmático foram encontrado como evidência de processamento anormal da dor em pacientes com FM, sendo semelhantes aos níveis encontrados em pacientes com distúrbios psiquiátricos, sendo uma possível explicação para as altas taxas de pacientes portadores de FM com Transtorno de Ansiedade Generalizada e Transtornos Depressivos.

A severidade dos distúrbios mentais foi vista ser paralela à severidade da FM, portanto quanto antes diagnosticada e tratada corretamente, com uma equipe multidisciplinar, menor será a severidade de distúrbios como depressão e ansiedade. Os estudos ainda citam, que o uso de antidepressivos ajudam a diminuir os sintomas depressivos e consequentemente os sintomas da FM, principalmente a dor, e podem propiciar melhor qualidade de vida ao paciente. As intervenções psicológicas, tem se mostrado altamente eficazes em todas as faixas etárias de 
pacientes com FM, entretanto, para adultos mais velhos é preciso um tratamento multifacetado para tratar, além da dor crônica causada pela FM, outras comorbidades que normalmente são encontradas nesses pacientes.

\section{Discussão}

Com base nos estudos, foi comprovada a intrínseca relação entre transtornos psíquicos com a fibromialgia, sendo porém muitas vezes difícil estabelecer a ordem cronológica do aparecimento dessas doenças. Alguns pacientes, relatam a fibromialgia como a doença de base com posteriori surgimento do transtorno psiquiátrico. Já alguns casos relatados, informam que o início desses dois transtornos foi concomitantemente.

\section{Conclusão}

Conclui-se que, os estudos analisam principalmente mulheres, acima dos 30 anos de idade, portadoras de Fibromialgia e devido a diversos fatores como, demora do diagnóstico, dificuldade de encontrar o tratamento correto, e dores crônicas, desenvolvem distúrbios mentais. Esses males comprometem a qualidade de vida desses pacientes, além de potencializarem as dores.

Pacientes com fibromialgia tem altas probabilidades de desenvolver alterações psicopatológicas, como depressão e ansiedade, sua vulnerabilidade à essas condições ocorrem devido à preocupação antecipatória, pessimismo e baixo autodirecionamento, sentimento de menor propósito e conformismo de sua condição, além de distúrbios de concentrações alteradas de neurotransmissores e hormônios, que corroboram a presença dos sintomas depressivos. 
1. Deborah Brown, et al. The relationship between psychological distress and multiple tender points across the adult lifespan. Archives of Gerontology and Geriatrics, Volume 63, 2016, [cited 2021, May 25] Available from: https://doi.org/10.1016/j.archger.2015.10.012

2. Lukkahatai, N., Walitt, B., Espina, A., Gelio, A. and Saligan, L.N. (2016), Understanding the Association of Fatigue With Other Symptoms of Fibromyalgia: Development of a Cluster Model. Arthritis Care \& Research, 68: 99-107. https://doi.org/10.1002/acr.22626

3. Roland van Rensburg, MBChB, Helgard Pieter Meyer, FCFP(SA), Sonia Anne Hitchcock, MPraxMed, Christian Edward Schuler, MMed (Psych), Screening for Adult ADHD in Patients with Fibromyalgia Syndrome, Pain Medicine, Volume 19, Issue 9, September 2018, Pages 1825-1831, https://doi.org/10.1093/pm/pnx275

4. Rosalind Gittins, MPharm, Molly Howard, PharmD, Ameer Ghodke, MS, Timothy J Ives, PharmD, MPH, Paul Chelminski, MD, MPH, FACP, The Accuracy of a Fibromyalgia Diagnosis in General Practice, Pain Medicine, Volume 19, Issue 3, March 2018, Pages 491-498, https://doi.org/10.1093/pm/pnx155

5. Attademo, L., \& Bernardini, F. (2018). Prevalence of personality disorders in patients with fibromyalgia: A brief review. Primary Health Care Research \& Development, 19(5), 523-528. doi:10.1017/S1463423617000871, https://doi.org/10.1017/S1463423617000871

6. Tonguc D. Berkol, Yasin H. Balcioglu, Simge S. Kirlioglu, Habib Erensoy and Meltem Vural Dissociative features of fibromyalgia syndrome. Neurosciences Journal July 2017, 22 (3) 198-204; DOI:https://doi.org/10.17712/nsj.2017.3.20160538

7. Coppens, E., Van Wambeke, P., Morlion, B., Weltens, N., Giao Ly, H., Tack, J., Luyten, P. and Van Oudenhove, L. (2017), Prevalence and impact of childhood adversities and 
post-traumatic stress disorder in women with fibromyalgia and chronic widespread pain. Eur J Pain, 21: 1582-1590. https://doi.org/10.1002/ejp.1059

8. Garcia-Fontanals, Alba $\mathrm{PhD}^{*}$; Portell, Mariona $\mathrm{PhD} \dagger$; García-Blanco, Susanna $\mathrm{PhD}$; Poca-Dias, Violant MD\$; García-Fructuoso, Ferran PhD, MDł; López-Ruiz, Marina MSc§; Gutiérrez-Rosado, Teresa PhD*; Gomà-i-Freixanet, Montserrat PhD*; Deus, Joan PhD*, Vulnerability to Psychopathology and Dimensions of Personality in Patients With Fibromyalgia, The Clinical Journal of Pain: November 2017 - Volume 33 - Issue 11 - p 991-997 doi: 10.1097/AJP.0000000000000506

9. Raneen Hellou, Winfried Häuser, Inbal Brenner, Dan Buskila, Giris Jacob, Ori Elkayam, Valerie Aloush, Jacob N. Ablin, "Self-Reported Childhood Maltreatment and Traumatic Events among Israeli Patients Suffering from Fibromyalgia and Rheumatoid Arthritis", Pain Research and Management, vol. 2017, Article ID 3865249, 8 pages, 2017. https://doi.org/10.1155/2017/3865249

10. Garcia-Fontanals, A., García-Blanco, S., Portell, M., Pujol, J., Poca-Dias, V., GarcíaFructuoso, F., López-Ruiz, M., Gutiérrez-Rosado, T., Gomà-i-Freixanet, M. and Deus, J. (2016), Cloninger's psychobiological model of personality and psychological distress in fibromyalgia. Int $\mathrm{J}$ Rheum Dis, 19: 852-863. https://doi.org/10.1111/1756$185 X .12473$

11. Brendon Stubbs, et al. A random effects meta-analysis investigating the prevalence of bipolar disorder in people with fibromyalgia: An updated analysis, Journal of Affective Disorders, Volume 191, 2016, Pages 308-309, Available from : https://doi.org/10.1016/j.jad.2015.12.006.

12. del Pozo-Cruz, J., Alfonso-Rosa, R.M., Castillo-Cuerva, A., Sañudo, B., Nolan, P. and del Pozo-Cruz, B. (2017), Depression symptoms are associated with key health outcomes in women with fibromyalgia: a cross-sectional study. Int J Rheum Dis, 20: 798-808. https://doi.org/10.1111/1756-185X.12564 
13. Gota, C.E., Kaouk, S. and Wilke, W.S. (2017), The impact of depressive and bipolar symptoms on socioeconomic status, core symptoms, function and severity of fibromyalgia. Int J Rheum Dis, 20: 326-339. https://doi.org/10.1111/1756-185X.12603

14. Simone Grassini \& Steven Nordin (2017) Comorbidity in Migraine with Functional Somatic Syndromes, Psychiatric Disorders and Inflammatory Diseases: A Matter of Central Sensitization?, Behavioral Medicine, 43:2, 91-99, DOI: $\underline{10.1080 / 08964289.2015 .1086721}$

15. Toussaint LL, Whipple MO, Vincent A. Post-traumatic stress disorder symptoms may explain poor mental health in patients with fibromyalgia. Journal of Health Psychology. 2017;22(6):697-706. doi:10.1177/1359105315611957 